\title{
MicroRNA-223 functions as an oncogene in human colorectal cancer cells
}

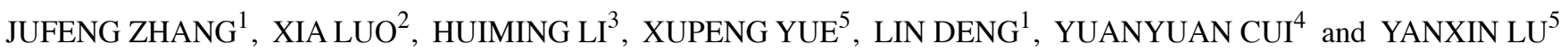 \\ ${ }^{1}$ School of Life Science and Biopharmaceutics, Guangdong Pharmaceutical University, Guangzhou, \\ Guangdong 510006; ${ }^{2}$ College of Engineering, South China Agricultural University, Guangzhou, Guangdong 510642; \\ ${ }^{3}$ Experimental Research Center, First People's Hospital, School of Medicine, Shanghai Jiaotong University, \\ Shanghai 200080, P.R. China; ${ }^{4}$ Department of Urology, UC Davis Medical Center, Sacramento, CA, \\ USA; ${ }^{5}$ Zhuhai Campus of Zunyi Medical College, Zhuhai, Guangdong 519041, P.R. China
}

Received January 20, 2014; Accepted March 28, 2014

DOI: $10.3892 /$ or.2014.3173

\begin{abstract}
Aberrant microRNA (miRNA) expression has been frequently observed in colorectal cancer (CRC), the third most common human cancer in the world. However, the roles of miRNAs in CRC remain poorly understood. The present study explored, identified and characterized the miRNAs that correlate with CRC progression. Deregulated level of microRNA-223 (miR-223) was screened out by miRNA microarray in colorectal tumor tissues and further confirmed by quantitative real-time PCR in a large cohort of CRC samples $(\mathrm{n}=90)$. After silencing miR-223 by artificial anti-miR-223 (miR-223-inhibitor), WST-1 and colony formation assays were employed to assess cell proliferation, and cell migration and invasion were evaluated by wound healing test and Transwell assays, respectively. Compared with adjacent non-tumor tissues, 22 miRNAs were screened out in CRC tissues with significance ( $>2$-fold), of which 13 were upregulated and 9 were downregulated. miR-223 is one of the noticeably upregulated miRNAs. Large cohort CRC sample analyses showed that a higher level of miR-223 correlated with a higher clinical stage. Reducing miR-223 expression resulted in a decreased cell proliferation, migration and invasion in CRC cells. miR-223 functions as an oncomiR in CRC, therefore serving as a potential diagnostic and therapeutic target for the treatment of CRC.
\end{abstract}

\section{Introduction}

Colorectal cancer (CRC) is the third most frequently diagnosed cancer worldwide with an increasingly high incidence

Correspondence to: Dr Yuanyuan Cui, Department of Urology, UC Davis Medical Center, Sacramento, CA, USA

E-mail: yuanyuan.cui@ucdmc.ucdavis.edu

Dr Yanxin Lu, Zhuhai Campus of Zunyi Medical College, Jinwan, Zhuhai, Guangdong 519041, P.R. China

E-mail: dream-nature@163.com

Key words: colorectal cancer, microRNA-223, oncogene rate in developing countries $(1,2)$. It is also the second leading cause of cancer-related mortality with very poor prognosis and a high possibility of metastasis $(3,4)$. To date, there is no ideal biomarker for CRC detection and therapy, therefore, development of biomarkers for early detection and therapy cannot be underrated, as they can help control progression and mortality of CRC.

MicroRNAs (miRNAs) are a class of non-coding small RNAs that can block the translation of mRNA targets and affect mRNA stability (5), thereby regulating protein-coding gene networks and pathways (6). Aberrant expressions of miRNAs have been observed in breast (7), brain (8), lung (9), thyroid $(10,11)$ and colon (11) cancer cells and are tightly related to cancer development and progression, including biological processes such as cellular proliferation, differentiation, apoptosis and development of metastases (12-14). miRNAs contribute to oncogenesis as either an oncogene (oncomiR) or a tumor-suppressor (15). In CRCs, miR-20a, miR-21, miR-25, miR-31, miR-93, miR-106, miR-183 and miR-203 were upregulated, suggesting their oncogene functions, whereas miR-1, miR-126, miR-30, miR-143, miR-145, miR-191 and miR-192 were downregulated, indicating their tumor suppressor functions (16). In CRCs, miRNAs are found to play an important role in cancer risk assessment, diagnosis, prognosis and drug response (17-19), therefore, they can serve as potential biomarkers. However, to date, only 50 miRNAs have been studied in CRC or CRC cell lines (16) and their functions are not well understood. Furthermore, there are few clinical analyses for the correlation between miRNAs and CRC progression.

MicroRNA-223 (miR-223) has been reported with opposite functions in different types of cancer. It functions as an oncomiR in some cancer types, such as recurrent vs. primary ovarian (20) and bladder cancer (21), adenocarcinoma of esophagus (22) and metastatic gastric cancer $(23,24)$, whereas in acute lymphoblastic leukemia (25), primary small cell lung cancer and hepatocellular carcinoma (26-28), miR-223 functions as a tumor suppressor. However, the function of miR-223 in CRC has yet to be characterized.

In the present study, we used miRNA microarray to compare the miRNA profiles in colon tumors and paired non-tumorous 
tissues. We identified a promising candidate, miR-223, and confirmed its expression pattern in CRC in 90 paired samples using quantitative real-time PCR. Furthermore, inhibition of miR-223 suppressed colon cancer cell proliferation, migration and invasion. Taken together, these results suggest that miR-223 plays a critical role in CRC and can function as a potential molecular biomarker and therapeutic gene for CRC treatment.

\section{Materials and methods}

Patients and samples. Pairs of human colon tumor and adjacent non-tumorous tissues were obtained from 90 surgical patients from the Department of Gastroenterology, Peking University Shenzhen Hospital. CRC samples were collected from patients undergoing bowel resection. Adjacent normal mucosa samples located at least $2 \mathrm{~cm}$ from the margins of the tumor (polyp or carcinoma) were used as controls. All tumors were adenocarcinomas. Cases with mucinous carcinomas (when $>50 \%$ of the tumor volume was composed of mucin) were excluded from this study. Collected samples were flash frozen in liquid nitrogen after surgery. All patients were informed about the aims of the specimen collection and they provided written consent in accordance with the ethics guidelines of Peking University. The present study was approved by the Ethical Committee of Peking University Shenzhen Hospital.

CRC cell culture and transfection. Human colorectal carcinoma cells LoVo and Colo320 were cultured in Dulbecco's minimum essential medium (DMEM) supplemented with $10 \%$ fetal bovine serum (FBS; Gibco). Artificial miR-223 inhibitor (anti-miR-223) and random sequence anti-miR molecule (anti-miR-NC) were synthesized by Ambion (Austin, TX, USA). LoVo and Colo320 cells were seeded for $20 \mathrm{~h}$ incubation before transfection followed by transfection with either chemically synthesized anti-miR-223 or anti-miR-NC using Lipofectamine 2000 (Invitrogen, Carlsbad, CA, USA) according to the manufacturer's instructions.

miRNA microarray. The miRNA microarray was performed at the Kangchen Biotechnology company (Shanghai, China). The LNA-modified oligonucleotide probes for all annotated miRNAs from human (Homo sapiens) in miRBase version 11.0 were purchased from Exiqon (miRCURY ${ }^{\mathrm{TM}}$ version 11.0). RNA labeling and hybridization on miRNA microarray chips was performed as previously described (29). Scanning of microarray was performed with an Axon GenePix 4000B microarray scanner. GenePix Pro V6.0 was used for image processing and array data retrieval. The intensity of green signal was calculated after background subtraction and replicated spots on the same slide were averaged to get the median intensity. The median normalization method was used to obtain 'Normalized Data'. Normalized Data $=($ Foreground - Background $) /$ median. Median was 50 percent quantile of miRNA intensity which was $>50$ in all samples after background correction. Unsupervised hierarchical clustering was performed on the miRNA expression profiling using cluster 3 and Treeview software.

Quantitative real-time PCR. Colorectal tumor and adjacent normal mucosa total RNA was extracted with TRIzol reagent
(Invitrogen). The expression of miR-223 was measured using TaqMan MicroRNA Assays kit (Ambion) according to the manufacturer's instructions. Relative expression was calculated by the $2^{-\Delta \Delta \mathrm{Ct}}$ method and normalized to the expression of RNU6B (Ambion).

Cell proliferation assays. The effect of miR-223 on the cell viability was measured by WST-1 assay. Cells were counted and plated at a density of $3 \times 10^{3}$ cells/well in 96-well plates in triplicates. Cell viability was determined at 24, 48 and $72 \mathrm{~h}$ post-transfection. Spectrophotometry was performed at $\lambda=450 \mathrm{~nm}$ and $\lambda_{\text {ref }}=630 \mathrm{~nm}$ after incubation with $10 \mu \mathrm{l} \mathrm{WST}-1$ (Roche, New York, NY, USA) for $2 \mathrm{~h}$. Proliferation of cells was evaluated using colony formation assay. For colony formation assays, cells were seeded in 6 -well plates $\left(0.5 \times 10^{3}\right.$ cells/well $)$ and cultured for two weeks. Colonies were fixed with methanol for $10 \mathrm{~min}$ and stained with $1 \%$ crystal violet (Sigma) for $1 \mathrm{~min}$. Visible colonies in 10 random view fields were manually counted. Each cell group was measured in triplicate.

Scratch wound-healing motility assays. Cells were seeded and grown to confluence in a 60-mm dish. An artificial wound was created $24 \mathrm{~h}$ following transfection with either anti-miR-223 or anti-miR-NC using a 200- $\mu$ l pipette tip on the confluent cell monolayer. To visualize migrated cells and wound healing, images were captured at 0 and $48 \mathrm{~h}$. The distance between the two edges of the scratch was measured using Digimizer software system.

Transwell invasion assay. Cell invasion assay was performed using the Matrigel Invasion Chamber (BD Biosciences, San Jose, CA, USA) pre-coated with ECM gel (Sigma, St. Louis, MO, USA). Cells transfected with either chemically synthesized anti-miR-223 or anti-miR-NC were seeded into the upper chamber of serum-free medium. The lower chamber prepared with $10 \%$ FBS served as a chemoattractant. After 24-h incubation, the non-invading cells were mechanically removed. The invasive cells on the lower surface of the membrane were then washed, fixed and stained with crystal violet. The number of cells was counted in 10 randomly optical fields under a microscope at a magnification of $\mathrm{x} 400$.

Statistical analysis. Significance analysis of microarrays (SAM) was used to identify miRNAs differentially expressed between samples $(\mathrm{FDR}=0)$. miR-223 expressions in $\mathrm{CRC}$ tumors and adjacent non-tumor tissues were compared by the Mann-Whitney U test and in the evaluation between early and advanced stage tumors. A comparison of means among two or more groups was performed using one-way analysis of variance or the Student's t-test. All numerical data were expressed as means \pm SD. P-value $<0.05$ was considered to indicate a statistically significant result. Statistical analyses were performed using GraphPad Prism 5.0 (GraphPad Software, San Diego, CA, USA) and SPSS software (version 11).

\section{Results}

Expression profiling of miRNAs in human CRC and adjacent non-tumor colorectal tissues. To investigate the miRNA expression patterns in human CRC, we used miRNA microar- 
A

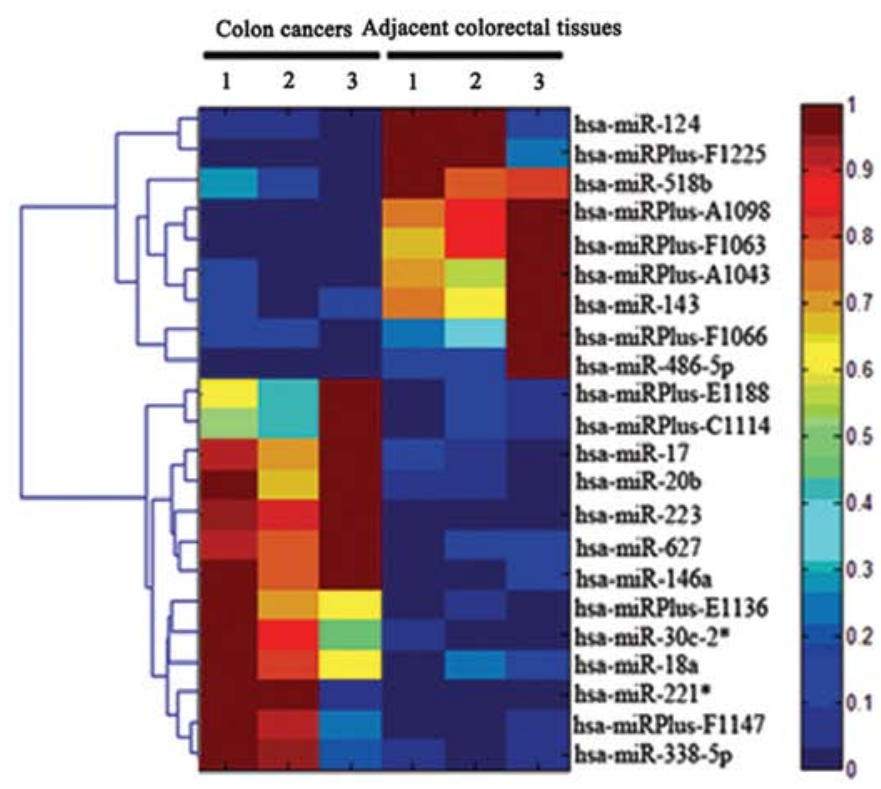

B

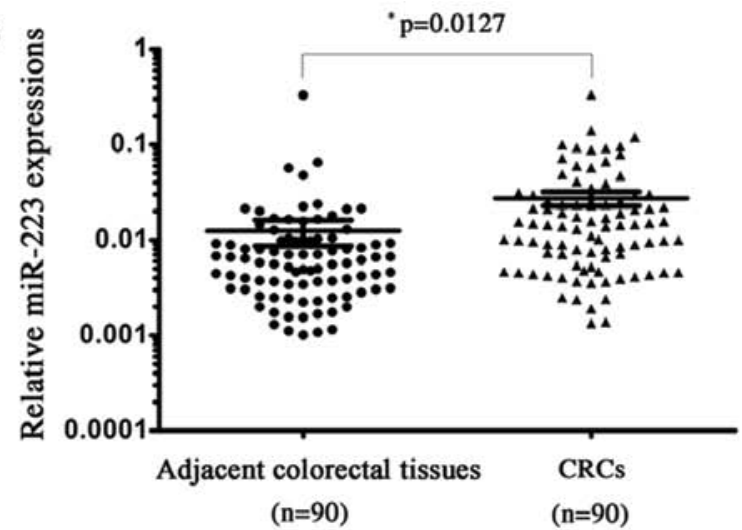

C

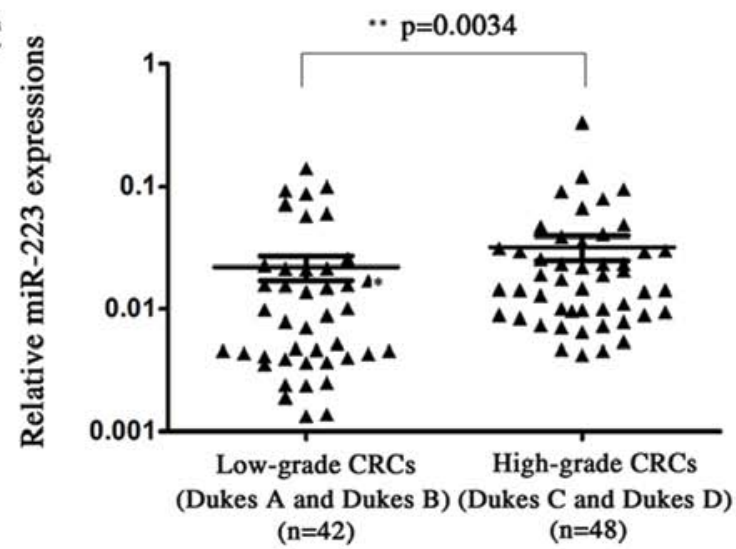

Figure 1. miR-223 is upregulated in human colorectal cancer (CRC). (A) Expression profiling of miRNAs in human CRC and adjacent non-tumor colorectal tissues. Total RNA isolated from three paired CRC and normal colorectal tissues were analyzed by miRNA array analysis. Hierarchical clustering analysis of significantly changed miRNAs was also shown. Relative changes in their expression level are indicated by a color code. Red indicates that the level of gene expression is higher than median, and blue indicates that the level is lower than median. Among these, miR-223 is one of the most frequently upregulated miRNAs in human CRC. (B) A significant upregulation of miR-223 was observed in CRC compared to adjacent non-malignant colorectal tissue ( $\mathrm{P}=0.0127)$. Total RNA isolated from 90 paired CRC and normal colorectal tissues were subjected to quantitative real-time PCR to validate differential expression of miR-223. Each assay was performed in triplicates and expression of miRNA was normalized to RNU6B. (C) A significant association of high miR-223 expression and high-grade CRCs was suggested $(\mathrm{P}=0.0034)$.

rays to compare their expression in human CRC and adjacent non-tumor colorectal tissues. Experiments were conducted with miRNA isolated from threepaired normal and tumor specimens. Microarray expression profiling revealed a series of miRNAs that showed altered expressions in CRC. Scatter plot and hierarchical clustering analysis are shown in Fig. 1A. Compared with adjacent non-tumor tissues, 13 miRNAs were upregulated for at least 2-fold, and 9 miRNAs were downregulated for at least 2-fold in CRCs. Upregulated miRNAs were miR-338-5p, miRPlus-C1114, miR-146a, miR-18a, miR-20b, miR-30c-2*, miRPlus-F1147, miR-17, miRPlus-E1136, miRPlus-E1188, miR-627, miR-221* and miR-223. Downregulated miRNAs were miR-486-5p, miRPlus-F1225, miR-124, miR-143, miR-518b, miRPlus-A1043, miRPlus-F1063, miRPlus-A1098 and miRPlus-F1066.

miR-223 is upregulated in human CRC. Since an interesting miRNA, miR-223, has been reported to be overexpressed in metastatic gastric cancer cells (30), we sought to analyze the function of miR-223 in CRC. To further confirm the upregulation of miR-223 in CRC, we performed quantitative real-time PCR in 90 paired CRC samples. The overexpression of miR-223 in CRC was identified with a significant P-value
( $\mathrm{P}=0.0127$; Fig. 1B). Furthermore, compared with $\mathrm{CRC}$ tissues from patients with low-grade CRC (Dukes A and B), high-grade (Dukes $C$ and $D$ ) patient tissues presented an even higher miR-223 ( $\mathrm{P}=0.0034$; Fig. 1C). These results suggest that miR-223 plays an important role in CRC progression and functions as a potential biomarker for early screening and diagnosis.

Suppressing miR-223 expression reduces CRC cell proliferation and viability. To further test the function of miR-223 in CRC progression, we use chemically synthesized miR-223 inhibitor (anti-miR-223) to downregulate miR-223 expression in human CRC cell lines, Colo320 and LoVo. Over $96 \mathrm{~h}$ after transfection of anti-miR-223 in both Colo320 and LoVo cells, the WST-1 absorbance at $450 \mathrm{~nm}$ subtracting background absorbance at $630 \mathrm{~nm}$ did not increase and almost remained unchanged whereas over 2-fold increase was observed in the anti-miR-NC transfected cells (Fig. 2A), suggesting a decreased proliferation and viability upon suppression of miR-223. Consistent with the results in WST-1 assay, cells transfected with anti-miR-223 showed significantly fewer colonies than cells transfected with anti-miR-NC $(\mathrm{P}<0.001)$ (Fig. 2B). 
A

B
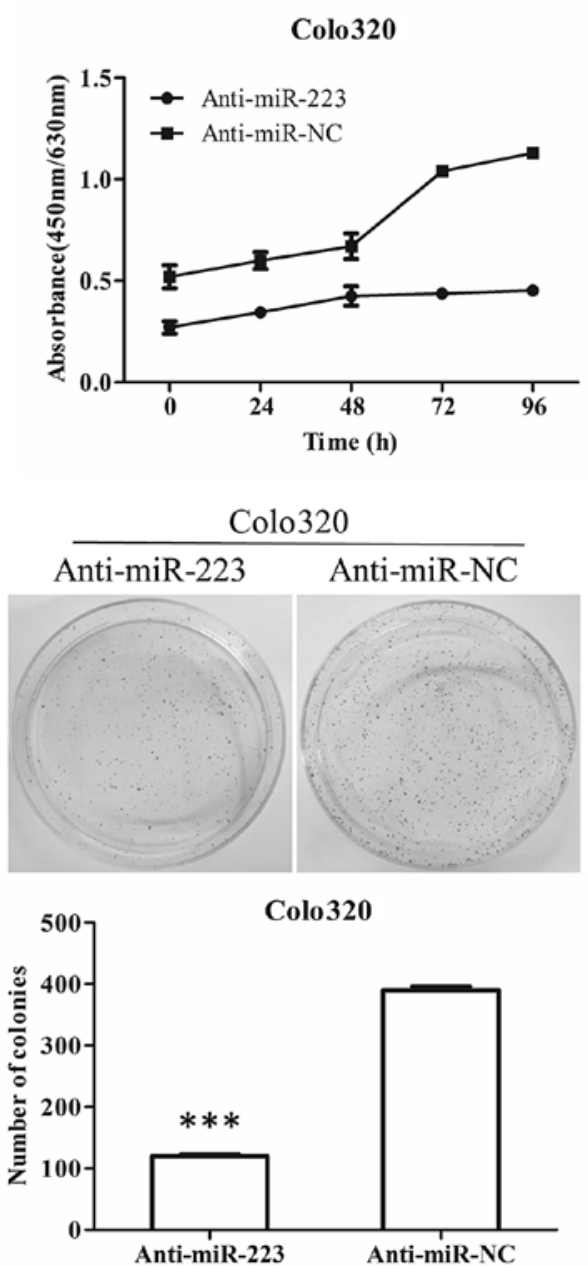

LoVo

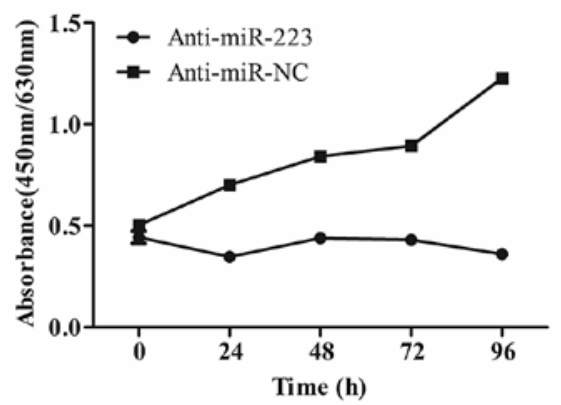

LoVo
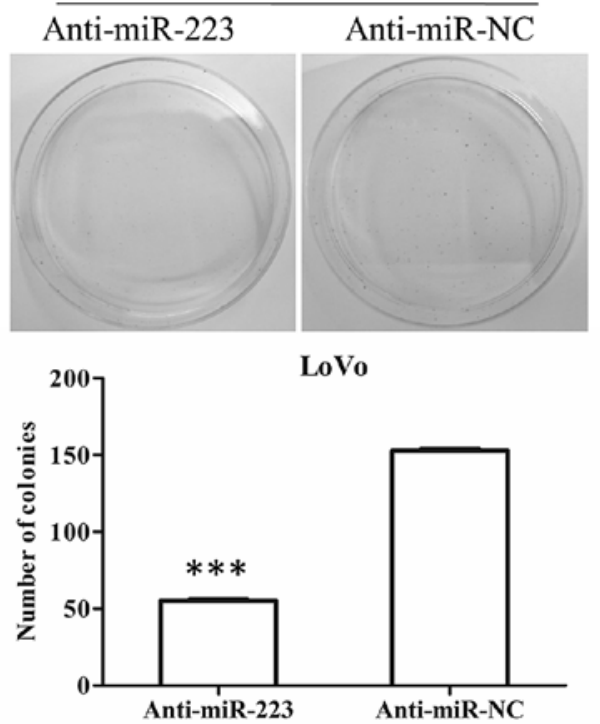

Figure 2. Colorectal cancer cell growth is suppressed by inhibiting miR-223. (A) WST-1 assay showed that Colo320/anti-miR-223 and LoVo/anti-miR-223 cells grew slower than cells transfected with the anti-miR-NC. (B) Colony formation assay of anti-miR-223 and anti-miR-NC-transfected Colo320 and LoVo cells. All data are shown as means $\pm \mathrm{SD}$. ${ }^{* * *} \mathrm{P}<0.001$.

Suppression of miR-223 inhibits migration and invasion of CRC cells. Since miR-223 has a higher level of tissues from high grade patients (Duke C and D) (Fig. 1C), suggesting a higher chance of metastasis, we used scratch wound healing assay and Transwell assay to evaluate cell migration and invasion ability.

Upon suppression of miR-223, both Colo320 and LoVo cells showed largely decreased motility and could not efficiently close the wound as anti-miR-NC transfected cells did over $48 \mathrm{~h}$ (Fig. 3). In addition to motility, after transfection of anti-miR-223, both Colo320 and LoVo cells showed less invasion ability through Matrigel coated Transwell compared with anti-miR-NC transfected cells (Fig. 4). These results suggest that suppression of miR-223 inhibits colon cancer cell migration and invasion.

\section{Discussion}

At present, although with a high mortality rate, upon detection of preneoplastic lesions with modern screening methods, the mortality and morbidity of CRC have modestly decreased and it has become a preventable disease (31). Yet, it is still insufficient to use present clinical and pathological parameters for early diagnosis or personalized treatment. As assessment of the microRNA (miRNA) genetic profile of colorectal cancer (CRC) and increasingly more miRNAs have been identified and characterized, they could serve as potential clinical biomarkers $(14,32)$.

The present study, for the first time, identified miR-223 and verified its oncogenetic function in CRC tissue. Using miRNA array and quantitative real-time PCR, we identified miR-223 as a highly upregulated miRNA in CRC compared to adjacent non-tumorous tissue (Fig. 1A and B). In addition, in tissues from higher grade (Duke C and D) patients, miR-223 levels were significantly higher than from lower grade (Duke A and B) patients (Fig. 1C). These findings led us to explore the functional analysis of miR-223. Downregulation of miR-223 was able to significantly suppress proliferation (Fig. 2), migration (Fig. 3) and invasion (Fig. 4) of colon cancer cells.

Other than miR-223, several other miRNAs with significant different expression between CRC and normal tissue were also identified by miRNA array (Fig. 1). Consistent with previous reports, the expression of miR-17, miR-18 and miR-20 was significantly higher in CRC (33-35). Functional analysis showed miR-17 increases proliferation, invasion and 
A
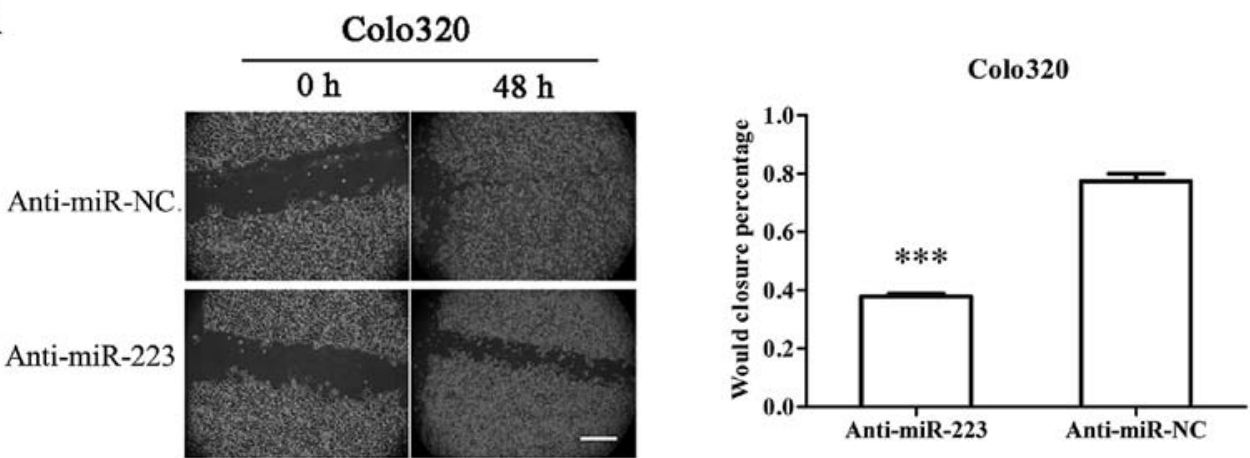

B
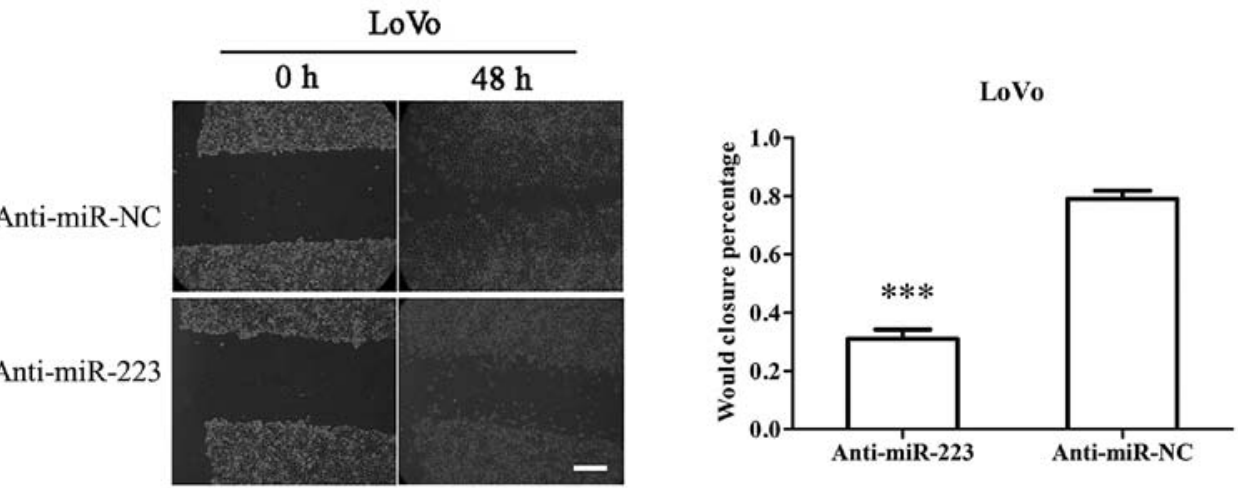

Figure 3. Knockdown of miR-223 reduces colorectal cancer cell migration. (A) Colo320 and (B) LoVo cells were transfected with anti-miR-223 or anti-miR-NC for $24 \mathrm{~h}$, and wounds were made. Magnification for assessment of migration is $\mathrm{x} 400$. Bar, $100 \mu \mathrm{m}$. The wound closure percentage per field is shown. All data are shown as means $\pm \mathrm{SD}$. ${ }^{* * *} \mathrm{P}<0.001$.

A

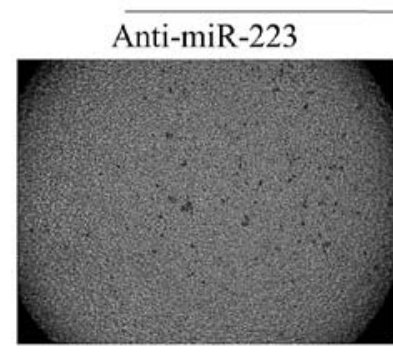

B

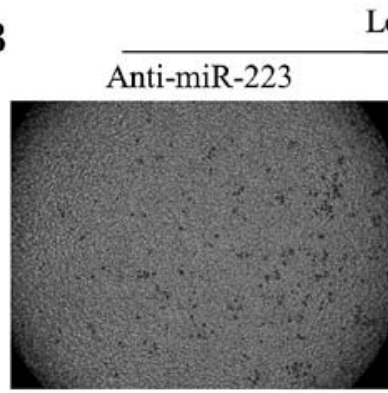

Colo320

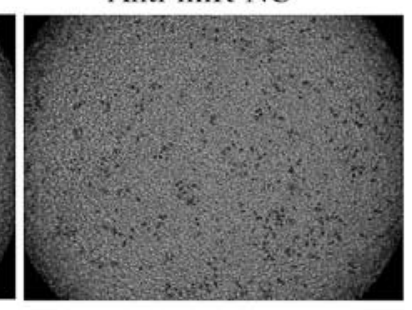

LoVo

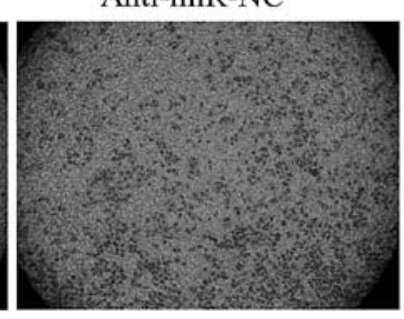

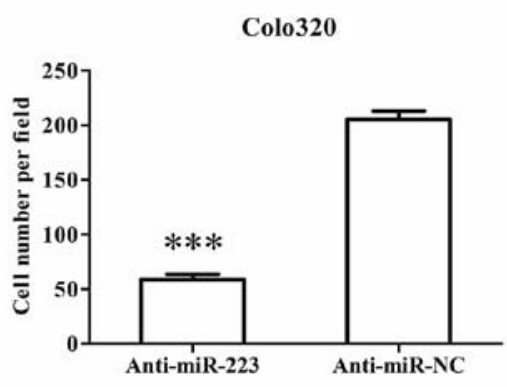

LoVo

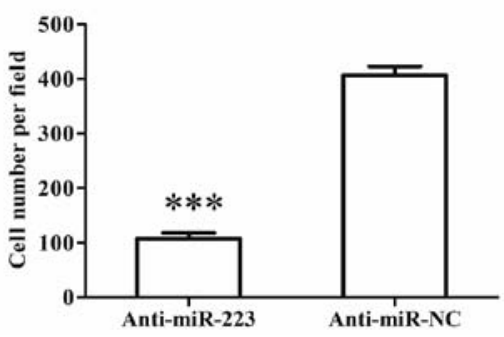

Figure 4. Inhibiting miR-223 decreases colorectal cancer cell invasion. (A) Colo320 and (B) LoVo cells were transfected with anti-miR-223 or anti-miR-NC for $24 \mathrm{~h}$, and Transwell invasion assay was performed. The invasive cells on the lower surface of the membrane were then washed, fixed and stained with crystal violet. The number of cells was counted in ten randomly optical fields under a microscope at a magnification of $\mathrm{x} 400$. All data are shown as means \pm SD. ${ }^{* * *} \mathrm{P}<0.001$.

migration of colon cancer cells $(34,35)$. On the other hand, miR-143 is downregulated in CRC tissue, and functions as an inhibitor for the cancer progression of CRC through multiple targets $(33,35-39)$.
By identifying these miRNAs, we could use miR-223 and other miRNAs as clinical biomarkers either by themselves or in combination with each other. It is possible that combining different miRNAs will give a more accurate clinical diagnosis. 


\section{Acknowledgements}

We thank our colleagues for their insight and technical support. This study was supported by the National Natural Scientific Foundation of China (grant no. 81171447), and the Natural Science Foundation of Guangdong Province, China (grant no. 8104518036002006310).

\section{References}

1. Siegel R, Ward E, Brawley O and Jemal A: Cancer statistics, 2011: the impact of eliminating socioeconomic and racial disparities on premature cancer deaths. CA Cancer J Clin 61: 212-236, 2011

2. Center MM, Jemal A and Ward E: International trends in colorectal cancer incidence rates. Cancer Epidemiol Biomarkers Prev 18: 1688-1694, 2009.

3. Ferlay J, Shin HR, Bray F, Forman D, Mathers C and Parkin DM: Estimates of worldwide burden of cancer in 2008: GLOBOCAN 2008. Int J Cancer 127: 2893-2917, 2010.

4. Edwards BK, Ward E, Kohler BA, et al: Annual report to the nation on the status of cancer, 1975-2006, featuring colorectal cancer trends and impact of interventions (risk factors, screening, and treatment) to reduce future rates. Cancer 116: 544-573, 2010

5. Winter J, Jung S, Keller S, Gregory RI and Diederichs S: Many roads to maturity: microRNA biogenesis pathways and their regulation. Nat Cell Biol 11: 228-234, 2009.

6. Bartel DP: MicroRNAs: genomics, biogenesis, mechanism, and function. Cell 116: 281-297, 2004.

7. Qu J, Zhao L, Zhang P, et al: MicroRNA-195 chemosensitizes colon cancer cells to the chemotherapeutic drug doxorubicin by targeting the first binding site of BCL2L2 mRNA. J Cell Physiol doi: 10.1002/jcp.24366, 2013 [Epub ahead of print].

8. Catania A, Maira F, Skarmoutsou E, D'Amico F, Abounader R and Mazzarino MC: Insight into the role of microRNAs in brain tumors (Review). Int J Oncol 40: 605-624, 2012.

9. Cherni I and Weiss GJ: miRNAs in lung cancer: large roles for small players. Future Oncol 7: 1045-1055, 2011.

10. Le Pennec S and Savagner F: MiRNAs in follicular thyroid tumors. Presse Med 40: 683-689, 2011 (In French).

11. Agostini M, Pucciarelli S, Calore F, Bedin C, Enzo M and Nitti D: miRNAs in colon and rectal cancer: a consensus for their true clinical value. Clin Chim Acta 411: 1181-1186, 2010.

12. Liu X, Zhang Z, Sun L, et al: microRNA-499-5p promotes cellular invasion and tumor metastasis in colorectal cancer by targeting FOXO4 and PDCD4. Carcinogenesis 32: 1798-1805, 2011.

13. He L and Hannon GJ: MicroRNAs: small RNAs with a big role in gene regulation. Nat Rev Genet 5: 522-531, 2004.

14. Croce CM: Causes and consequences of microRNA dysregulation in cancer. Nat Rev Genet 10: 704-714, 2009.

15. Lu J, Getz G, Miska EA, et al: MicroRNA expression profiles classify human cancers. Nature 435: 834-838, 2005.

16. Corte H, Manceau G, Blons H and Laurent-Puig P: MicroRNA and colorectal cancer. Dig Liver Dis 44: 195-200, 2012.

17. Schetter AJ, Leung SY, Sohn JJ, et al: MicroRNA expression profiles associated with prognosis and therapeutic outcome in colon adenocarcinoma. JAMA 299: 425-436, 2008.

18. Boni V, Zarate R, Villa JC, et al: Role of primary miRNA polymorphic variants in metastatic colon cancer patients treated with 5-fluorouracil and irinotecan. Pharmacogenomics J 11: 429-436, 2011.

19. Landi D, Gemignani F, Naccarati A, et al: Polymorphisms within micro-RNA-binding sites and risk of sporadic colorectal cancer Carcinogenesis 29: 579-584, 2008.
20. Laios A, O'Toole S, Flavin R, et al: Potential role of miR-9 and miR-223 in recurrent ovarian cancer. Mol Cancer 7: 35, 2008.

21. Gottardo F, Liu CG, Ferracin M, et al: Micro-RNA profiling in kidney and bladder cancers. Urol Oncol 25: 387-392, 2007.

22. Mathé EA, Nguyen GH, Bowman ED, et al: MicroRNA expression in squamous cell carcinoma and adenocarcinoma of the esophagus: associations with survival. Clin Cancer Res 15: 6192-6200, 2009

23. Li X, Zhang Y, Zhang $\mathrm{H}$, et al: miRNA-223 promotes gastric cancer invasion and metastasis by targeting tumor suppressor EPB41L3. Mol Cancer Res 9: 824-833, 2011.

24. Kang W, Tong JH, Chan AW, et al: Stathmin1 plays oncogenic role and is a target of microRNA-223 in gastric cancer. PLoS One 7: e33919, 2012.

25. Mi S, Lu J, Sun M, et al: MicroRNA expression signatures accurately discriminate acute lymphoblastic leukemia from acute myeloid leukemia. Proc Natl Acad Sci USA 104: 19971-19976, 2007.

26. Miko E, Czimmerer Z, Csánky E, et al: Differentially expressed microRNAs in small cell lung cancer. Exp Lung Res 35: 646-664, 2009.

27. Karakatsanis A,Papaconstantinou I, Gazouli M,Lyberopoulou A, Polymeneas G and Voros D: Expression of microRNAs, miR-21, miR-31, miR-122, miR-145, miR-146a, miR-200c, miR-221, miR-222, and miR-223 in patients with hepatocellular carcinoma or intrahepatic cholangiocarcinoma and its prognostic significance. Mol Carcinog 52: 297-303, 2013.

28. Wong QW, Lung RW, Law PT, et al: MicroRNA-223 is commonly repressed in hepatocellular carcinoma and potentiates expression of Stathminl. Gastroenterology 135: 257-269, 2008.

29. Sempere LF, Christensen M, Silahtaroglu A, et al: Altered MicroRNA expression confined to specific epithelial cell subpopulations in breast cancer. Cancer Res 67: 11612-11620, 2007.

30. Xing J, Wan S, Zhou F, et al: Genetic polymorphisms in premicroRNA genes as prognostic markers of colorectal cancer. Cancer Epidemiol Biomarkers Prev 21: 217-227, 2012.

31. Lieberman DA: Clinical practice. Screening for colorectal cancer. N Engl J Med 361: 1179-1187, 2009.

32. Iorio MV and Croce CM: MicroRNA dysregulation in cancer: diagnostics, monitoring and therapeutics. A comprehensive review. EMBO Mol Med 4: 143-159, 2012.

33. Motoyama K, Inoue H, Takatsuno Y, et al: Over- and underexpressed microRNAs in human colorectal cancer. Int $\mathrm{J}$ Oncol 34: 1069-1075, 2009.

34. Zhang J, Xiao Z, Lai D, et al: miR-21, miR-17 and miR-19a induced by phosphatase of regenerating liver-3 promote the proliferation and metastasis of colon cancer. Br J Cancer 107: 352-359, 2012.

35. Monzo M, Navarro A, Bandres E, et al: Overlapping expression of microRNAs in human embryonic colon and colorectal cancer. Cell Res 18: 823-833, 2008.

36. Liu H, Zhu L, Liu B, et al: Genome-wide microRNA profiles identify miR-378 as a serum biomarker for early detection of gastric cancer. Cancer Lett 316: 196-203, 2012.

37. Slaby O, Svoboda M, Fabian P, et al: Altered expression of miR-21, miR-31, miR-143 and miR-145 is related to clinicopathologic features of colorectal cancer. Oncology 72: 397-402, 2007.

38. Ng EK, Tsang WP, Ng SS, et al: MicroRNA-143 targets DNA methyltransferases $3 \mathrm{~A}$ in colorectal cancer. $\mathrm{Br} \mathrm{J}$ Cancer 101: 699-706, 2009.

39. Chen X, Guo X, Zhang H, et al: Role of miR-143 targeting KRAS in colorectal tumorigenesis. Oncogene 28: 1385-1392, 2009. 